\title{
Oral Verrucous Carcinoma: A Study of 12 Cases
}

\author{
Alper Alkana \\ Emel Bulut ${ }^{\mathrm{b}}$ \\ Omer Gunhanc \\ Bora Ozden ${ }^{b}$
}

\section{ABSTRACT}

Objectives: The purpose of this clinical study was to identify a clinical and histopathological relationship between verrucous hyperplasia, verrucous keratosis, and verrucous carcinoma.

Methods: We evaluated 12 patients who had developed oral verrucous carcinoma in the past 10 years in a follow-up study. In this study, the diagnostic criteria included clinical and histopathological features of the lesions. Each lesion was examined by a single oral pathologist.

Results: All the patients were diagnosed with verrucous carcinoma following excisional biopsy. One patient was diagnosed with verrucous hyperplasia and another with verrucous keratosis in their initial histological findings. Mandibular, posterior alveolar crest, and retromolar trigone were the most affected sites (41.6\%), followed by the buccal mucosa (16.6\%), the palate $(16.6 \%)$, the floor of the mouth (16.6\%), and the lip (8.3\%). No patients had evidence of recurrence after treatment.

Conclusions: Verrucous hyperplasia, verrucous keratosis, and verrucous carcinoma may not be distinguished clinically or may coexist, resulting in diagnostic difficulties. It should be kept in mind that verrucous hyperplasia may also develop from leukoplakic lesions, and it may transform into verrucous carcinoma or squamous-cell carcinoma, acting as a potential precancerous lesion. (Eur J Dent 2010;4:202-207)

Key words: Oral; Verrucous carcinoma; Verrucous hyperplasia; Verrucous keratosis.

- associate Professor, Department of Oral and Maxillofacial Surgery, Faculty of Dentistry, University of Erciyes, Kayseri, Turkey.

${ }^{b}$ Assistant Professor, Department of Oral and Maxillofacial Surgery, Faculty of Dentistry, University of Ondokuz Mayis, Samsun, Turkey.

Professor, Department of Pathology,

Gulhane Military Medical Academy, Ankara, Turkey.

- Corresponding author: Dr. Emel Bulut Ondokuz Mayis Universitesi, Dis Hekimligi Fakultesi 55139 Kurupelit, Samsun, Turkey.

Phone: +90 362 3121919-3289

Fax: +903624576032

E-mail: euzundaomu.edu.tr

\section{INTRODUCTION}

Oral verrucous carcinoma is a rare tumor first described by Ackerman. ${ }^{1}$ It is a special form of well-differentiated squamous cell carcinoma with specific clinical and histological features. Various names are used in the literature to describe this entity, including Ackerman's tumor, BuschkeLoewenstein tumor, florid oral papillomatosis, epithelioma cuniculatum, and carcinoma cuniculatum. ${ }^{2}$ The tumor grows slowly and locally, invasive in nature and unlikely to metastasize. It appears as a painless, thick white plaque resem- 
bling a cauliflower. The most common sites of oral mucosal involvement include the buccal mucosa, followed by the mandibular alveolar crest, gingiva, and tongue. Shear and Pindborg ${ }^{3}$ described a condition termed verrucous hyperplasia in 1980. Both lesions closely resemble each other clinically and pathologically. Verrucous hyperplasia has been considered an antecedent stage or early form of verrucous carcinoma and is believed to have the same biological potential. 4,5 Surgery has been the first choice of treatment for these lesions, and radiotherapy is controversial; ${ }^{6}$ however, surgery combined with radiotherapy is the next most preferable treatment and may have benefits, particularly in cases of extensive lesions. ${ }^{7}$ Recurrence rate is high in cases in which either irradiation or surgery alone is performed.

The purpose of this article is to describe 12 cases of oral verrucous carcinoma with an analysis of the literature and to identify the clinical and histopathological relationship between verrucous carcinoma and its similar pathological entities.

\section{MATERIALS AND METHODS}

A search of the files of the Department of Oral and Maxillofacial Surgery, Samsun, and Department of Pathology, Gulhane Military Medical Academy, Ankara, between 1995 and 2005 revealed 12 cases of verrucous carcinoma. The diagnostic criteria in the present study included clinical and histopathological features of the lesions. Each lesion was examined by a single oral pathologist.

\section{RESULTS}

The clinical details are summarized in Table 1. Among the 12 patients lage range: 42-77, mean age: $58.3,7$ males and 5 females in a ratio of 1.4:1 in sequence), lesions affected a variety of intraoral sites. The posterior alveolar crest and retromolar trigone were the most affected sites (41.6\%), followed by the buccal mucosa $(16.6 \%)$, the palate $(16.6 \%)$, the floor of the mouth $(16.6 \%)$, and the lip mucosa $(8.3 \%)$, respectively.

Patients' medical history revealed no systemic diseases. All the lesions were cauliflower-shaped exophytic lesions clinically (Figure 1). One patient also had a leukoplakic area extending to the cheek mucosa associated with the lesion (Figure 2). Four patients were smokers of cigarettes alone. Among these patients, one (patient no. 10) had smoked cigarettes for 10 years (more than one pack a day), three had been smoking for 40 years (more than two packs a day). In the study, two patients were using alcohol and cigarettes together. One of these patients had smoked cigarettes and drunk alcohol together for 40 years, while the other one had consumed alcohol for 10 years and smoked cigarettes for 30 years. One patient had trauma prosthesis, 2 patients had no possible etiologic factors, and sufficient records were not found for 2 patients.

No regional lymph node involvement was found in an extraoral examination of all cases. An incisional biopsy was taken from all lesions, and the specimens were examined histopathologically. In initial histological findings, one patient was consistent with verrucous hyperplasia and another with verrucous keratosis. For definitive differential diagnosis, the entire masses with their surrounding tissues had to be excised. Verrucous carcinoma was diagnosed following histopathological examination of the excisional biopsy specimens. General histopathological characteristics of the excised specimens revealed acanthosis, papillomatosis, and hyperkeratosis of the epithelium of the lesion, continuing with characteristics of healthy mucosa. Squamous epithelial cell composition of the tumors did not give a definite atypical character, but showed blunt rete processes toward the subepithelial area. Lymphocyte infiltration was noted in the periphery of the tumor islands (Figure 3). In all patients, no recurrences were observed in a 2-year follow-up (Figure 4).

Only one patient received radiotherapy and a cytostatic agent after surgical treatment (Table 1); however, xerostomia related to radiotherapy had developed that affected that patient's daily life. To relieve the symptoms, the patient was advised to take saliva substitutes.

\section{DISCUSSION}

Schrader et $\mathrm{al}^{8}$ and Jordan ${ }^{9}$ have reported that verrucous carcinomas were slow-growing, exophytic, well-demarcated hyperkeratotic lesions. These typically present as extensive, white, warty lesions. ${ }^{9}$ All the lesions in this case series were similar in clinical behavior and aspect.

The etiology of verrucous carcinoma is not well defined. Human papillomavirus (HPV) has been considered one of the causative factors. ${ }^{10}$ Smok- 
ing seems highly associated with the development of mucosal verrucous carcinoma of the neck and head. Poor oral hygiene, presence of oral lichenoid, and leukoplakic lesions may act as predisposing factors. In Asia, leukoplakia is known to be associated with smoking (bidis and cigarettes), smokeless tobacco, and chewing habits (paan, areca quid, and miang), and a synergistic effect has also been found. ${ }^{11}$ In Lee et al's study, ${ }^{12}$ subjects who had chewed one or more betel quids or had smoked one or more cigarettes per day for at least 1 year were defined as ever chewers or ever smokers. Subjects who had drunk one or more bottles of alcoholic beverage (including beer, liquor, and winel per month for at least 1 year were defined as ever drinkers. Among them, current users were those who had practiced these habits within the past year, and ex-users were those who

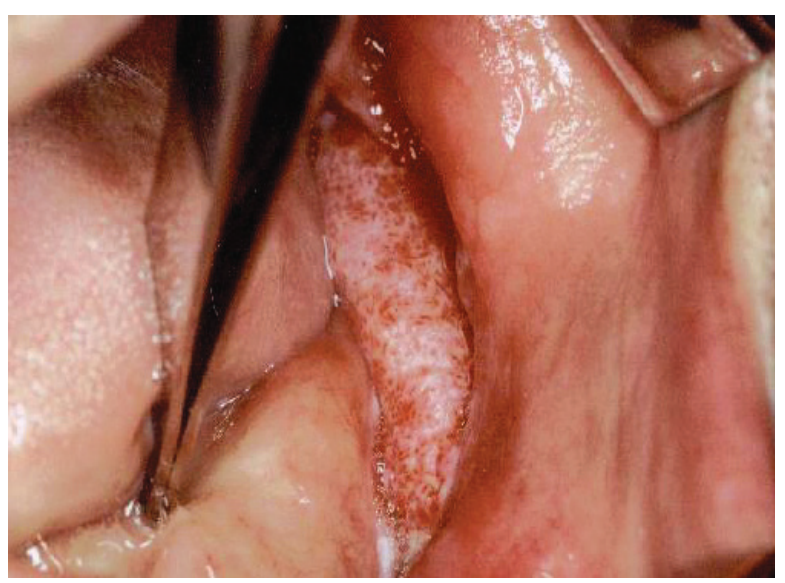

Figure 1. Intraoral aspect of the mass exhibiting cauliflower shape. had stopped the habit for at least 1 year before diagnoses or interviews. For all the ever chewers and ever smokers, a detailed history of their chewing and smoking habits was recorded, including daily consumption, age of commencement, and duration of practice. For ever drinkers, information on frequency of alcohol intake was collected. To assess the cumulative risks of betel quid chewing and cigarette smoking, the number of "packyears," calculated by multiplying the number (in packs; 20 cigarettes or 10 betel quids per pack) consumed daily by years of use, was employed as an indicator of chewing and smoking. In addition, the types of regularly chewed betel materials were recorded, as follows: areca nut with a piece of inflorescence of Piper Betel Linn, areca nut with a piece of betel leaf, and both mixed. Their results suggested that, although betel quid chewing was

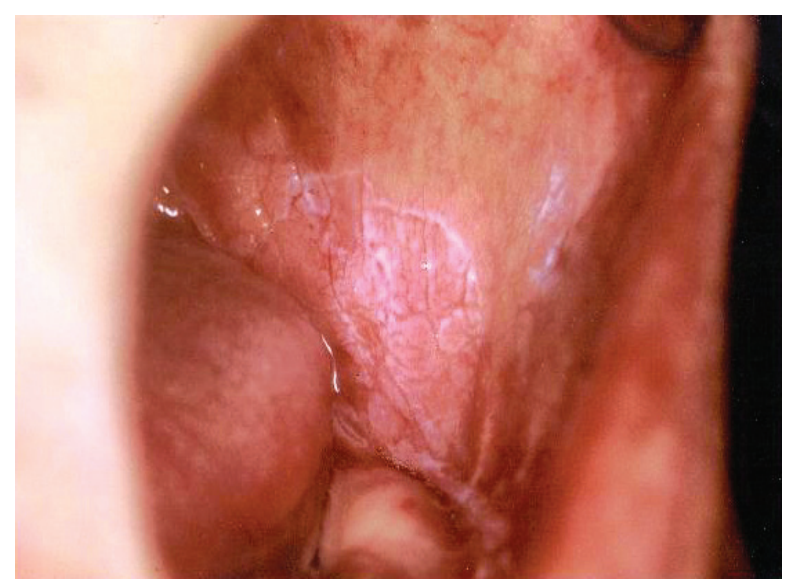

Figure 2. Leukoplakic area extending to the cheek mucosa.

Table 1. Summary of the 12 patients with verrucous carcinoma.

\begin{tabular}{|c|c|c|c|c|c|}
\hline No & Age & Sex & Therapy & Location & Possible etiologic factor \\
\hline 1 & 73 & M & Surg, X-Rad & Mandible, PAC & Leukoplakia \\
\hline 2 & 68 & M & Surg & Floor of the mouth & Cigarette smoking \\
\hline 3 & 55 & $\mathrm{~F}$ & Surg & Hard palate & Trauma of prosthesis \\
\hline 4 & 77 & $\mathrm{~F}$ & Surg & Mandible, PAC & Cigarette smoking and alcohol \\
\hline 5 & 70 & $\mathrm{~F}$ & Surg & Lip mucosa & Cigarette smoking \\
\hline 6 & 42 & $\mathrm{~F}$ & Surg & Retromolar trigone & Unknown etiology \\
\hline 7 & 47 & $\mathrm{~F}$ & Surg & Retromolar trigone & No record available \\
\hline 8 & 47 & M & Surg & Hard palate & Cigarette smoking and alcohol \\
\hline 9 & 47 & M & Surg & Retromolar trigone & No record available \\
\hline 10 & 50 & M & Surg & Buccal mucosa & Cigarette smoking \\
\hline 11 & 58 & M & Surg & Buccal mucosa & Cigarette smoking \\
\hline 12 & 65 & M & Surg & Floor of the mouth & Unknown etiology \\
\hline
\end{tabular}

Abbreviations: M: Male, F: Female, Surg: Surgery, X-Rad: Radiotherapy PAC: Posterior Alveoler Crest 
a major cause of both oral leukoplakia and oral submucous fibrosis, its effect on the two diseases might be different. Cigarette smoking has a modifying effect on development of oral leukoplakia. The composition and method of chewing may vary widely from country to country. In Turkey, in contrast to some countries, chewing of betel nut or areca quid is not a habit; cigarette smoking is generally preferred. Alcohol consumption alone or in conjunction with tobacco usage also increases the risk of carcinogenesis. ${ }^{13}$ A case-control study in Taiwan showed that the odds ratio was 123 times greater for patients with oral cancer who smoked, drank alcohol, or chewed areca quid than for nonusers. Precancerous disorders are known to be associated with cigarette smoking, excess alcohol consumption, and areca quid chewing among Asians. ${ }^{11}$

Shear and Pindborg ${ }^{3}$ reported that out of 28 patients with verrucous lesions, 24 (86\%) used tobacco, and one was an areca quid chewer. Tobacco appears to be a major factor in causation of verrucous lesions. In Chen et al's ${ }^{14}$ study of verrucous carcinomas in Taiwan, areca quid chewing

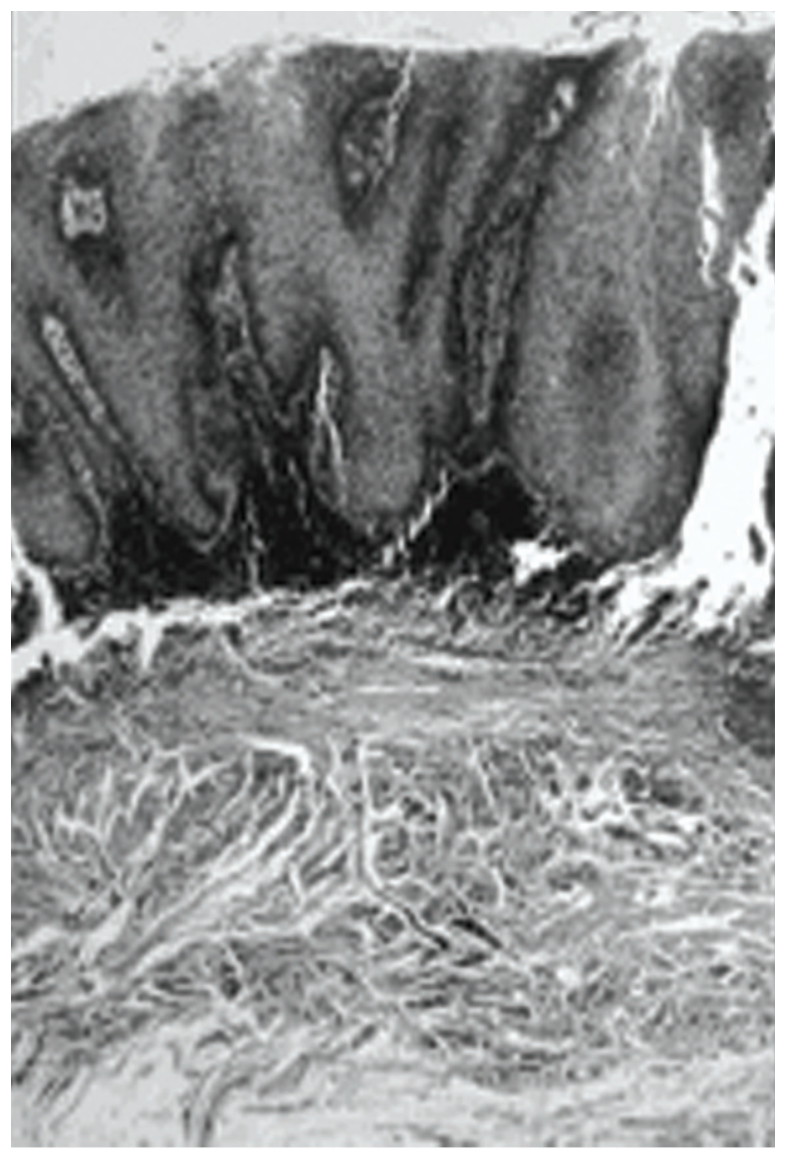

Figure 3. Histopathological appearance of verrucous carcinoma (HE x 50). was reported by $97.3 \%$. In Chung et $\mathrm{al}^{\prime} \mathrm{s}^{11}$ study, the prevalence of verrucous lesions (not carcinomas) was $0.84 \%$, and the frequency of current areca quid chewing in this subgroup was $55.6 \%$ (5/9). The data indicate that, in Taiwan, areca quid use could be a major risk factor in verrucous lesions. In our patients, cigarette smoking seemed the most causative factor among those mentioned above.

Verrucous hyperplasia and verrucous carcinoma are indistinguishable clinically. ${ }^{4,7}$ The clinical association with leukoplakia is significant, and the evidence indicates that untreated leukoplakia may develop into a verrucous hyperplasia and/or a verrucous carcinoma in time. Leukoplakia, in fact, is a clinical provisional designation for a keratotic white mucosal lesion whose diagnosis cannot be ascertained on clinical grounds, and that therefore requires a biopsy. The biopsy can reveal any one of many possible diagnoses, including a benign keratosis, a precancerous epithelial lesion such as chronic candidosis, and lichen planus. Shear and Pindborg ${ }^{3}$ noted that 36 of 68 patients had verrucous hyperplasia associated with leukoplakic lesions. Similarly, our study revealed one patient having verrucous hyperplasia accompanied by leukoplakic lesions. It is often difficult to distinguish between verrucous hyperplasia and verrucous carcinoma. Verrucous hyperplasia is a forerunner of verrucous carcinoma, and transition is so consistent that the hyperplasia, once diagnosed, should be treated as verrucous carcinoma. ${ }^{10}$ Verrucous hyperplasia warrants close clinical follow-up to intercept and prevent such a possibility. Differential diagnosis can be made histologically, but a biopsy specimen should be suffi-

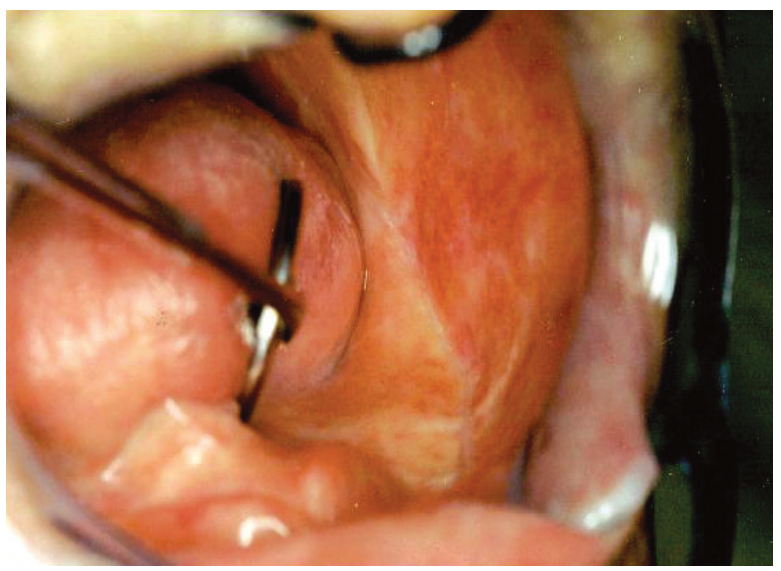

Figure 4. Appearance of the healing area 2 years postoperatively.

\section{April 2010 - Vol.4}


cient for correct diagnosis. Verrucous hyperplasia generally does not extend into deeper tissues but is superficial to normal epithelium, whereas verrucous carcinoma extends more deeply. ${ }^{3,4,7}$ In one of our cases, the preliminary diagnosis was verrucous hyperplasia following the incisional biopsy, whereas final diagnosis was verrucous carcinoma following the excisional biopsy. Another patient was diagnosed with verrucous keratosis in initial biopsy, but the final diagnosis of all patients was verrucous carcinoma. In our cases, histopathologic appearance was concurrent with those mentioned in the literature.

In spite of the 4:1 female/male ratio in Hansen et al's ${ }^{15}$ study and an approximately equal sex distribution in other studies, in our study, this ratio was 1.4:1 male/female.

In the current literature, ${ }^{16}$ although the most common site for verrucous carcinoma is the buccal mucosa, the most affected areas in the present study were the mandibular retromolar and molar area $(41.6 \%)$ followed by the buccal mucosa $(16.6 \%)$, the hard palate $(16.6 \%)$, the floor of the mouth $(16.6 \%)$, and the lip mucosa $(8.3 \%)$.

In verrucous carcinoma, regional lymph nodes are often tender and enlarged because of inflammatory involvement, simulating metastatic tumor. ${ }^{17}$ Contrarily, lymph nodes were not affected in our cases.

Verrucous carcinoma typically has a heavily keratinized, or parakeratinized, irregular clefted surface with parakeratin extending deeply into the clefts. The prickle cell layers show bulbous hyperplasia, but, for a considerable time at least, the tumor has a well-defined lower border and basal lamina. Atypia is minimal, and there is usually a subepithelial inflammatory infiltrate. ${ }^{17,18}$ Our cases presented histopathological findings similar to those mentioned above.

The prognosis of verrucous carcinoma is better than that of other kinds of life-threating malignant tumors. Surgery is considered the primary mode of treatment for verrucous carcinoma. Irradiation alone or in combination with surgery is rarely performed. Combined therapy can be useful when the tumor extends to the retromolar area. McClure et $\mathrm{al}^{7}$ reported that extensive lesions in the oral cavity may benefit from combined therapy. When surgery is not indicated, other treatment modalities such as cytostatic drugs may be preferred. Vari- ous dosages of cytostatic drugs have been proven to show beneficial effects in reducing tumor size; a-interferon (IFN) seems to support the therapy by delaying the growth of the tumor but does not take the place of surgery alone. ${ }^{19}$ Only one patient in our study received radiotherapy and a cytostatic agent after surgical treatment, since this patient's tumor extended to the retromolar area; however, xerostomia related to radiotherapy had developed and affected that patient's daily life. To relieve the symptoms, the patient was advised to use saliva substitutes. The other patients were treated with surgery. None of the patients had evidence of recurrence in 2 years of follow-up.

\section{CONCLUSIONS}

Verrucous hyperplasia, verrucous keratosis, and verrucous carcinoma may not be distinguished clinically or may coexist. It should be kept in mind that verrucous hyperplasia may also develop from leukoplakic lesions, and it may transform into verrucous carcinoma or squamous-cell carcinoma, acting as a potential precancerous lesion. Two of our cases were initial histopathological misdiagnoses; one was verrucous keratosis and the other was verrucous hyperplasia. In fact, all our cases were verrucous carcinoma. Thus both clinicians and pathologists must be careful about warty and exophytic lesions in the oral cavity.

\section{REFERENCES}

1. Ackerman LV.Verrucous carcinoma of oral cavity. Surgery 1948;23:670-678

2. Schwartz RA. Verrucous carcinoma of the skin and mucosa. J Am Acad Dermatol 1995;32:1-21.

3. Shear M, Pindborg JJ. Verrucous hyperplasia of the oral mucosa. Cancer 1980;46:1855-1862.

4. Murrah VA, Batsakis JG. Proliferative verrucous leukoplakia and verrucous hyperplasia. Ann Otol Rhinol Laryngol 1994;103:660-663.

5. Regezi JA, Sciubba J. Oral Pathology. Clinical-pathologic correlations. Philadephia, WB: Saunders Company, 1993.

6. Yoshimura Y, Mishima K, Obara S, Nariaib Y, Yoshimura H, Mikami T. Treatment modalities for oral verrucous carcinomas and their outcomes: contribution of radiotherapy and chemotherapy. Int J Clin Oncol 2001;6:192-200.

7. McClure DL, Gullane PJ, Slinger RP, Wysocki GP. Verrucous carcinoma-changing concepts in management. J Otolaryngol 1984;13:7-12. 
8. Schrader M, Laberke HG, Jahnke K. Lymphatic metastases of verrucous carcinoma (Ackerman tumor). HNO 1987;35:27-30.

9. Jordan RC. Verrucous carcinoma of the mouth. J Can Dent Assoc 1995;61:797-801.

10. Eversole LR. Papillary lesions of the oral cavity: relationship to human papillomaviruse. J Calif Assoc 2000;28:922927.

11. Chung $\mathrm{CH}$, Yang $\mathrm{YH}$, Wang TY, Shieh TY, Warnakulasuriya S. Oral precancerous disorders associated with areca quid chewing, smoking, and alcohol drinking in southern Taiwan. J Oral Pathol Med 2005;34:460-466.

12. Lee CH, Ko YC, Huang HL, Chao YY, Tsai CC, Shieh TY, Lin LM. The precancer risk of betel quid chewing, tobacco use and alcohol consumption in oral leukoplakia and oral submucous fibrosis in southern Taiwan. Br J Cancer 2003;88:366-372.

13. Koch BB, Trask DK, HoffmanHT, Karnell LH, Robinson RA, Zhen W, et al. National Survey of head and neck verrucous carcinoma. Cancer 2001;92:110-120.

14. Chen BL, Lin CC, Chen $\mathrm{CH}$. Oral verrucous carcinoma: an analysis of 73 cases. Clin J Oral Maxillofac Surg 2000;11:1117.

15. Hansen LS, Olson JA, Silverman S. Proliferative verrucous leukoplakia: a long term study of thirty patients. Oral Surg Oral Med Oral Pathol 1985;60:285-298.

16. Yeh CJ. Treatment of verrucous hyperplasia and verrucous carcinoma by shave excision and simple cryosurgery. Int $J$ Oral Maxillofac Surg 2003;32:280-283.

17. Shafer WG, Hine MK, Levy BM. Benign and malign tumors of the oral cavity In: A Textbook of Oral Pathology. Philadelphia, WB: Saunders Company; 1983. p. 127-130.

18. Cawson RA, Binnie WH, Speight PM, Barrett AW, Wright JM. Uncommon types of carcinoma. In: Lucas's Pathology of Tumors of the Oral Tissues. London: Churchill Livigstone; 1998. p. 241-242.

19. Risse L, Negrier P, Dang PM, Bedane C,Bernard P, Labrousse $F$, et al. Treatment of verrucous carcinoma with recombinant alfa-interferon. Dermatology 1995;190:142144. 